\title{
Surveillance des expositions en laboratoire aux agents pathogènes humains et aux toxines au Canada en 2017
}

\author{
D Pomerleau-Normandin'1 , M Heisz ${ }^{1}, \mathrm{~F}^{1}$ Tanguay ${ }^{1 *}$
}

\section{Résumé}

Contexte : En vertu de la Loi sur les agents pathogènes humains et les toxines et de son Règlement, l'Agence de la santé publique du Canada (ASPC) est chargée de surveiller les déclarations d'incidents en laboratoire par l'intermédiaire du système de surveillance de déclaration des incidents en laboratoire au Canada (DILC). L'année 2017 représente la deuxième année complète de données.

Objectif : Décrire les incidents d'exposition en laboratoire et les infections contractées en laboratoire survenus au Canada en 2017, selon le secteur, les agents pathogènes humains et les toxines en cause, le nombre de personnes touchées, le type d'incident et les causes fondamentales.

Méthodologie : Les incidents pris en compte dans la présente analyse sont survenus entre le $1^{\mathrm{er}}$ janvier et le 31 décembre 2017. Ils ont été déclarés à l'ASPC par des laboratoires titulaires de permis en vigueur, par I'intermédiaire du système de surveillance de DILC. Le programme Microsoft Excel 2010 a été utilisé pour les statistiques descriptives de base.

Résultats : Au total, 44 incidents liés à des expositions et à des infections contractées en laboratoire ont été signalés au moyen du système de surveillance de DILC en 2017. D'après la comparaison par secteur et proportion respective de permis, le nombre d'incidents était le plus élevé dans les secteurs académique et hospitalier comparativement aux laboratoires gouvernementaux et à l'industrie privée. En tout, 118 personnes ont été exposées, ce qui représente une moyenne de 2,7 personnes par incident (intervalle de 1 à 29). Aucune exposition secondaire n'a été signalée. Six incidents d'exposition (14\%) ont donné lieu à des cas "soupçonnés " $(n=5)$ ou confirmés $(n=1)$ d'infection contractée en laboratoire. Même si, dans I'ensemble, les agents pathogènes humains et les toxines du groupe de risque (GR) 2 étaient impliqués dans la majorité des incidents $(n=23 ; 52 \%)$, Francisella tularensis $(n=4 ; 9 \%)$ et Coccidioides immitis $(n=3 ; 7 \%)$ ont été le plus fréquemment mis en cause dans les incidents d'exposition déclarés. Ces deux agents pathogènes sont à la fois des agents biologiques du GR3 et des agents biologiques à cote de sécurité élevée (ABCSE). Chaque incident a été attribué à 2,3 causes fondamentales en moyenne $(n=101)$. Les problèmes relatifs aux procédures opérationnelles normalisées (PON) et l'erreur humaine ont été les deux causes les plus fréquentes.

Conclusion : La fréquence des incidents d'exposition en laboratoire a été relativement faible en 2017. La voie d'exposition la plus courante était l'inhalation, tandis que les causes fondamentales les plus fréquentes étaient les problèmes relatifs aux PON et l'erreur humaine. Par ailleurs, ce système de surveillance étant nouveau, les estimations de base demeurent en cours d'établissement.

Citation proposée : Pomerleau-Normandin D, Heisz M, Tanguay F. Surveillance des expositions en laboratoire aux agents pathogènes humains et aux toxines au Canada en 2017. Relevé des maladies transmissibles au Canada 2018;44(11):337-44 https://doi.org/10.14745/ccdr.v44i11a05f

Mots-clés : expositions en laboratoire, incidents de laboratoire, infections contractées en laboratoire, agents pathogènes humains, surveillance, bactéries, virus, toxines, biosécurité

\author{
Affiliation \\ ${ }^{1}$ Centre de la biosûreté, Agence \\ de la santé publique du Canada, \\ Ottawa (Ontario)
}

\section{*Correspondance :}

florence.tanguay@canada.ca 


\section{Introduction}

Les laboratoires qui permettent l'étude et le diagnostic d'agents pathogènes et de leurs toxines posent un risque d'exposition inhérent pour le personnel qui les manipule. Pourtant jusqu'à récemment, les incidents en laboratoire étaient uniquement signalés à l'interne et, le cas échéant, aux autorités responsables de la santé au travail. Quelques pays ont défini des exigences nationales de déclaration des incidents liés à la biosûreté et à la biosécurité (1-3). Cependant, c'est l'Agence de la Santé publique du Canada (ASPC) qui a établi l'un des premiers systèmes complets et normalisés de surveillance des incidents en laboratoire mettant en cause des agents pathogènes humains et des toxines à l'échelle nationale. Le système de surveillance de DILC a été mis en service en décembre 2015, en réponse aux exigences établies par la Loi sur les agents pathogènes humains et les toxines (LAPHT) de 2009 (4) et le Règlement sur les agents pathogènes humains et les toxines (RAPHT) (5) qui a suivi. L'année 2017 représente donc la deuxième année complète de données recueillies par l'intermédiaire du système de surveillance de DILC.

En vertu de la LAPHT et du RAPHT, les organisations doivent, à moins d'en avoir été exemptées, détenir un permis les autorisant à exercer des activités réglementées avec des agents pathogènes humains et des toxines. Fait à souligner, une organisation peut posséder plusieurs permis, et un seul permis peut couvrir plusieurs zones de confinement. La grande majorité des activités menées en laboratoire au Canada porte sur des agents pathogènes humains et des toxines $(93,2 \%)$ du groupe de risque (GR) 2, lesquels posent un risque modéré pour les personnes, mais un risque faible pour la santé publique, car ils peuvent causer des maladies graves mais peu probables chez I'humain. Une minorité d'activités en laboratoire (6,4 \%) porte sur des agents pathogènes humains du GR3, lesquels posent un risque élevé pour les personnes, mais faible pour la santé publique, car s'ils peuvent causer des maladies graves chez I'humain, ils sont peu susceptibles de se propager. Le travail de laboratoire avec des organismes du GR4, lesquels posent le risque individuel et collectif le plus élevé, représente seulement $0,2 \%$ de toutes les activités réglementées. De même, les activités faisant intervenir des agents biologiques à cote de sécurité élevée (ABCSE) ne représentent que 0,2\% de toutes les activités réglementées au Canada. Les ABCSE constituent un sous-ensemble d'agents pathogènes humains et de toxines « qui présentent un risque accru en raison de la possibilité qu'on les utilise comme arme biologique » $(6,7)$. Consulter l'annexe pour connaître la définition de certains termes d'usage courant.

La LAPHT impose aux établissements titulaires d'un permis de déclarer à I'ASPC les incidents mettant en cause des agents pathogènes humains et des toxines du GR2 ou d'un niveau de risque supérieur. Les déclarations visent les incidents avec ou sans exposition. Les expositions sont définies comme un contact ou une proximité étroite avec des agents pathogènes humains ou des toxines pouvant causer une intoxication ou une infection contractée en laboratoire (ICL). Quant aux incidents sans exposition, ils concernent la possession, la production ou la libération accidentelle d'un agent pathogène humain ou d'une toxine, ou la disparition, la perte ou le vol d'un agent pathogène ou d'une toxine, ou un ABCSE qui n'est pas reçu par son destinataire dans les 24 heures à compter du moment prévu (8). La première année complète de données provenant du système de surveillance de DILC remonte à 2016 (9). La présente étude porte sur les incidents d'exposition survenus en 2017.

Ce rapport vise à décrire les incidents d'exposition en laboratoire survenus au Canada entre le 1er janvier et le 31 décembre 2017, selon le secteur, les agents pathogènes humains et les toxines en cause, le nombre de personnes touchées, le type d'incident et ses causes fondamentales.

\section{Méthodologie}

Le système de surveillance de DILC fait appel à une interface personnalisée du programme de gestion des relations avec la clientèle de Microsoft Dynamics (Microsoft Corporation, Redmond, Washington, États-Unis). Les données sont entrées au moyen de formulaires normalisés propres au type de déclaration soumise; la plupart des champs de données dans ces formulaires sont obligatoires, ce qui permet des comparaisons précises et exactes. Même si les incidents sont autodéclarés, l'exactitude des déclarations est validée tout au long d'un processus d'enquête; l'information peut être mise à jour jusqu'à ce que le rapport de suivi final soit terminé et soumis en ligne par le déclarant. II importe de noter que plusieurs rapports de suivi peuvent être soumis pour un seul événement. Dans la présente étude, nous avons utilisé les données du rapport de suivi final. Les incidents considérés comme hors du champ d'application de la LAPHT et du RAPHT ont été exclus de l'analyse.

Le rapport de déclaration initial fournit les éléments essentiels liés à l'incident, tels que des renseignements administratifs et une brève description de l'incident. Quant au rapport de suivi, il contient de l'information sur les résultats de l'enquête, les personnes touchées et les mesures correctives adoptées.

Les données des rapports sur les expositions et les infections contractées en laboratoire (soupçonnées ou confirmées) en 2017 ont été extraites du système après s'être assuré que toutes les données prévues pour ladite année avaient été saisies. Les $I C L$ sont souvent confirmées dans le rapport de suivi d'après les résultats de l'enquête. Certains cas ne sont toutefois jamais confirmés et demeurent des cas "soupçonnés ". Par exemple, s'il est impossible d'écarter la possibilité que l'infection ait pu être contractée à l'extérieur de la zone de confinement (c.-à-d. d'origine communautaire), alors I'ICL demeurera « soupçonnée ». En fin de compte, le statut de l'ICL est déterminé d'après l'évaluation des risques et l'enquête menées sur les lieux. 
Les éléments de données issus des rapports d'exposition initiaux et utilisés pour l'analyse comprennent les renseignements sur le permis (nombre de permis et secteur [académique, hospitalier, privé/entreprises, santé publique, vétérinaire/ santé animale, environnement, etc.]) et les dates importantes (date de l'incident, date de la déclaration à l'autorité interne et date de la déclaration initiale à l'ASPC). Les éléments de données provenant du rapport de suivi et utilisés pour l'analyse regroupent le type d'incident, les renseignements sur les personnes touchées (nombre de cas primaires, nombre de cas secondaires, voie d'exposition, premiers soins, pharmacothérapie et prophylaxie post-exposition), la date de soumission du rapport de suivi, l'agent biologique en cause (type et groupe de risque) et la ou les causes fondamentales de l'incident.

Le programme Microsoft Excel 2010 (Microsoft Corporation, Redmond, Washington, États-Unis) a été utilisé pour l'analyse descriptive de base. Toutes les données sur les expositions et les ICL ont été déclarées, sauf lorsqu'elles pouvaient mener à I'identification exacte de l'établissement titulaire d'un permis. Le cas échéant, l'information a été exclue du présent rapport pour des raisons de sécurité et de confidentialité.

\section{Résultats}

Au 31 décembre 2017, 905 permis en vigueur autorisant des activités réglementées comportant la manipulation d'agents pathogènes humains ou de toxines ont été délivrés au Canada. Entre le 1er janvier et le 31 décembre 2017, les laboratoires titulaires d'un permis en vigueur ont transmis 51 déclarations d'incidents d'exposition au système de surveillance de DILC. À la suite du processus d'enquête, l'exposition a été écartée dans 7 cas, laissant un total de 44 incidents d'exposition. L'échantillon comportait trois incidents dont la date de survenue exacte n'avait pas pu être déterminée, mais les circonstances (type d'incident et date de signalement aux autorités internes) ont permis de conclure qu'ils s'étaient produits en 2017. Au total, $4,9 \%$ de toutes les installations titulaires de permis ont fait l'objet d'incidents d'exposition ou d'infection contractée en laboratoire.

Tous les cas confirmés d'exposition ( $n=44,100 \%)$ sont survenus dans des laboratoires de niveau de confinement 2 (NC2).

Dans une majorité de cas, il s'agissait d'incidents d'exposition seulement ( $n=38 ; 86 \%$ ). Parmi les six cas $d^{\prime} I C L$, cinq sont demeurés "soupçonnés ", et un seul a donné lieu à une ICL confirmée (figure 1).

\section{Incidents d'exposition par secteur}

La figure 2 compare le nombre de permis en vigueur $(N=905)$ au nombre de déclarations d'exposition $(\mathrm{N}=44)$ transmises au système de surveillance de DILC par secteur en 2017. Cette même année, les laboratoires ayant signalé le plus grand nombre d'incidents d'exposition provenaient des secteurs académique et hospitalier. Pourtant, comparativement à la proportion respective
Figure 1 : Sélection de cas et incidents d'exposition retenus pour l'analyse survenus au Canada en 2017

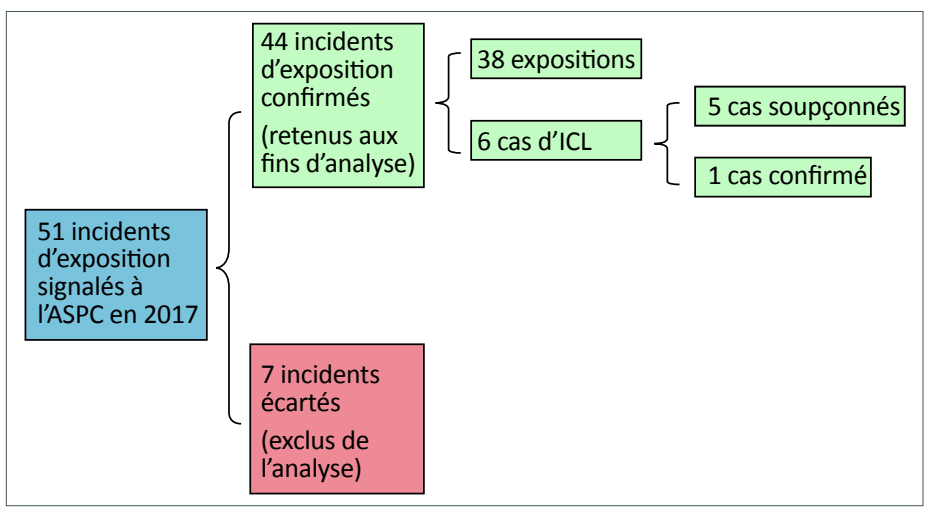

Abréviations : ICL, intoxication ou infection contractée en laboratoire; ASPC, Agence de la Santé publique du Canada

de permis détenus dans ces secteurs, la fréquence des incidents d'exposition était plus élevée dans les secteurs de la santé publique $(20 \%)$ et hospitalier (8,6\%). C'est dans le secteur privé/ entreprises et dans les autres secteurs gouvernementaux que la plus faible fréquence des incidents d'exposition a été observée,

Figure 2 : Permis en vigueur et incidents d'exposition à des agents pathogènes humains ou à des toxines signalés par secteur au Canada en 2017

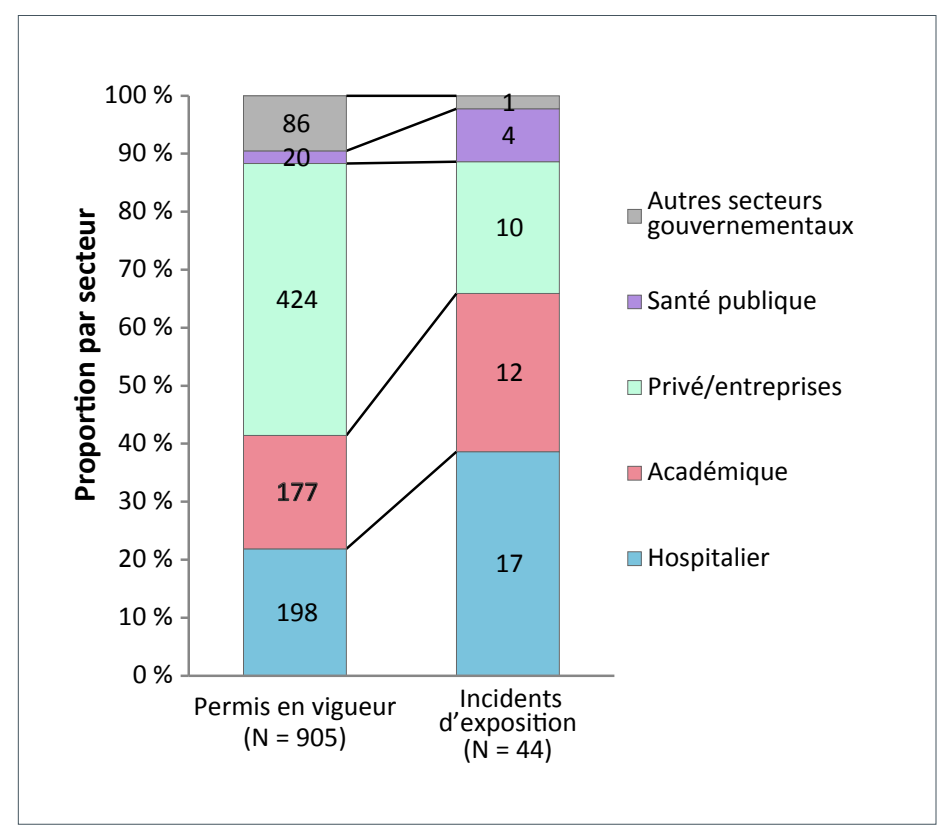

Abréviation : N, nombre total

Notes : Les données proviennent du système de surveillance de DILC (Canada, données extraites le 23 mars 2018)

Le secteur " académique » comprend notamment les universités, les collèges de médecine vétérinaire, les collèges et les cégeps [établissements et collèges techniques pré-universitaires québécois financés par l'État] et d'autres établissements d'enseignement Le secteur « hospitalier » regroupe les hôpitaux affiliés et non affiliés à une université La catégorie "secteur privé/entreprises » comprend les industries de la santé animale, de la sante humaine et de la biotechnologie, l'industrie pharmaceutique, l'industrie alimentaire, ainsi que les fournisseurs d'agents pathogènes et de toxines

Le secteur de la "santé publique » comprend le gouvernement fédéral, les gouvernements provinciaux et territoriaux et les administrations municipales

Les « autres secteurs gouvernementaux » regroupent les laboratoires vétérinaires ou de santé animale, les laboratoires environnementaux et d'autres laboratoires fédéraux, provinciaux, territoriaux et municipaux 
ces incidents s'étant produits respectivement dans $2,4 \%$ et $1,2 \%$ des établissements titulaires de permis.

\section{Agents pathogènes humains et toxines en cause}

Le tableau 1 présente la distribution de chaque agent pathogène humain en fonction de sa cote de sécurité (ABCSE ou non-ABCSE), du groupe de risque de la toxine (2, 3 ou 4) et du type d'organisme (bactérie, virus, toxine, prion ou inconnu) mentionnés. Des agents pathogènes humains ou des toxines du GR2 étaient en cause dans la majorité des incidents $(n=23$; $52 \%$ ). Des agents pathogènes humains ou des toxines du GR3 étaient en cause dans 14 incidents (32\%). L'agent pathogène humain ou la toxine en cause n'a pas pu être identifié(e) dans 7 incidents (16\%). Parmi les incidents avec des agents pathogènes humains ou des toxines ( $n=37$ ), les bactéries étaient le plus souvent en cause ( $n=21 ; 57 \%)$, suivies des virus ( $n=10 ; 27 \%)$.

Tableau 1 : Agents pathogènes humains ou toxines en cause dans les incidents d'exposition survenus au Canada en 2017, selon le groupe de risque de l'agent biologique

\begin{tabular}{|l|r|r|r|r|r|r|}
\hline \multirow{2}{*}{$\begin{array}{c}\text { Agent } \\
\text { biologique } \\
\text { par groupe de } \\
\text { risque }\end{array}$} & \multicolumn{2}{|c|}{ Non-ABCSE } & \multicolumn{2}{c|}{ ABCSE } & \multicolumn{2}{c|}{ Total } \\
\cline { 2 - 7 } & \multicolumn{1}{|c|}{$\%$} & \multicolumn{1}{c|}{$\mathbf{n}$} & \multicolumn{1}{c|}{$\%$} & \multicolumn{1}{c|}{$\mathbf{n}$} & \multicolumn{1}{c|}{$\%$} \\
\hline GR2 & 22 & 88 & 1 & 8 & 23 & 52 \\
\hline Bactérie & 11 & 44 & 0 & - & 11 & 25 \\
\hline Toxine & 1 & 4 & 1 & 8 & 2 & 5 \\
\hline Virus & 10 & 40 & 0 & - & 10 & 23 \\
\hline GR3 & 3 & 12 & 11 & 92 & 14 & 32 \\
\hline Bactérie & 2 & 8 & 8 & 67 & 10 & 23 \\
\hline Champignon & 0 & - & 3 & 25 & 3 & 7 \\
\hline Prion & 1 & 4 & 0 & - & 1 & 2 \\
\hline Inconnu & 0 & - & 0 & - & 7 & 16 \\
\hline Total & 25 & 100 & 12 & 100 & 44 & 100 \\
\hline
\end{tabular}

Abréviations : $n$, nombre, $\mathrm{ABCSE}$, agents biologiques à cote de sécurité élevée;

GR, groupe de risque; -, sans objet

Notes : Les données proviennent du système de surveillance de DILC. Les activités nécessitant la manipulation de matières biologiques du GR1 ne sont pas réglementées par la LAPHT. Les chiffres sont arrondis au nombre entier le plus près

Les 44 incidents d'exposition ont donné lieu à l'identification de 25 types différents d'agents pathogènes humains et de toxines. Les trois organismes les plus souvent mis en cause dans les déclarations d'incidents d'exposition étaient Francisella tularensis ( $n=4 ; 9 \%)$, Coccidioides immitis $(n=3 ; 7 \%)$ - tous deux des ABCSE du GR3 - et Salmonella spp ( $n=3 ; 7 \%$ ).

\section{Nombre de personnes touchées}

En tout, 118 personnes ont été exposées lors des 44 incidents d'exposition ou d'infection contractées en laboratoire. Le nombre de personnes exposées par incident en 2017 se situait entre un et 29. La moyenne était de 2,7 et la médiane de 1,0.
Dans la majorité des incidents d'exposition ( $n=33 ; 75 \%)$, une seule personne a été exposée. Les incidents impliquant l'exposition de plus de 10 personnes $(n=3)$ ont été signalés par les secteurs privé/entreprises $(n=1)$ et hospitalier $(n=2)$. L'incident impliquant l'exposition de 29 personnes s'est produit dans un laboratoire de diagnostic et était lié à la croissance lente d'une culture de Brucella réalisée dans un milieu de culture standard et manipulée au cours de plus d'un quart de travail. Le tableau 2 présente les agents pathogènes associés aux cas d'exposition et d'infection contractée en laboratoire

\section{Tableau 2 : Nombre d'incidents en laboratoire et de} personnes exposées, selon le groupe de risque et le type d'agents pathogènes humains, au Canada en 2017

\begin{tabular}{|c|c|c|c|c|}
\hline $\begin{array}{l}\text { Agent } \\
\text { biologique }\end{array}$ & $\begin{array}{l}\text { Incidents } \\
(N=44)\end{array}$ & $\begin{array}{l}\text { Personnes } \\
\text { exposées } \\
(n=118)\end{array}$ & $\begin{array}{c}\mathrm{ICL} \\
\text { soupçonnées } \\
(n=5)\end{array}$ & $\begin{array}{c}\text { ICL } \\
\text { confirmées } \\
(n=1)\end{array}$ \\
\hline GR2 & 23 & 25 & 3 & 1 \\
\hline $\begin{array}{l}\text { Virus de la } \\
\text { rubéole }\end{array}$ & 2 & 4 & - & - \\
\hline Salmonella spp & 3 & 3 & 2 & - \\
\hline $\begin{array}{l}\text { Escherichia coli } \\
\text { O157:H7 }\end{array}$ & 1 & 1 & - & 1 \\
\hline $\begin{array}{l}\text { Virus de la } \\
\text { Vaccine }\end{array}$ & 2 & 1 & 1 & - \\
\hline $\begin{array}{l}\text { Autres } \\
\text { organismes du } \\
\text { GR2 }\end{array}$ & 15 & 16 & - & - \\
\hline GR3 & 14 & 85 & 1 & 0 \\
\hline Brucella suis & 1 & 29 & - & - \\
\hline $\begin{array}{l}\text { Francisella } \\
\text { tularensis }\end{array}$ & 4 & 23 & - & - \\
\hline Brucella abortus & 1 & 19 & - & - \\
\hline $\begin{array}{l}\text { Coccidioides } \\
\text { immitis }\end{array}$ & 3 & 4 & - & - \\
\hline $\begin{array}{l}\text { Mycobacterium } \\
\text { spp }\end{array}$ & 2 & 4 & 1 & - \\
\hline $\begin{array}{l}\text { Autres } \\
\text { organismes du } \\
\text { GR3 }\end{array}$ & 3 & 6 & - & - \\
\hline Inconnu & 7 & 8 & 1 & 0 \\
\hline Total & 44 & 118 & 5 & 1 \\
\hline
\end{tabular}

Plus du quart des personnes touchées ( $n=34 ; 29 \%$ ) ont reçu une prophylaxie post-exposition dans les sept jours suivant I'incident. En outre, 16 personnes (14\%) ont reçu des premiers soins (6\%) et sept ont reçu un traitement pharmacologique. Aucun cas de transmission secondaire à l'ICL n'a été signalé.

\section{Types d'incidents}

La figure 3 présente les types d'incidents d'exposition déclarés en 2017 et les activités ou événements qui ont mené à ces incidents. La plupart des déclarations portaient sur des 
incidents liés à des procédures inadéquates ou à des bris de procédure $(n=13 ; 30 \%)$ et à des objets pointus ou tranchants $(n=13 ; 30 \%)$. Parmi les 44 incidents d'exposition déclarés, $14(32 \%)$ étaient également liés à la possession accidentelle d'un agent biologique du GR3 dans un laboratoire de niveau de confinement 2 (données non affichées). Un tel scénario est plus fréquent dans les laboratoires de diagnostic, car les échantillons reçus peuvent contenir des agents pathogènes ou des toxines non identifiées. Parmi les 14 cas de possession accidentelle, 11 (79\%) ont été signalés dans les secteurs de la santé publique et hospitalier. Les expositions liées à la possession accidentelle ont souvent mis en cause des agents pathogènes humains ou des toxines pouvant se transmettre par voie aérogène (p. ex., lors de la manipulation de Brucella spp, Burkholderia pseudomallei, Coccidioides immitis et Francisella tularensis).

Figure 3 : Types d'agent pathogène humain ou de toxine en cause dans les incidents d'exposition signalés au Canada en 2017 ( $N=60)$

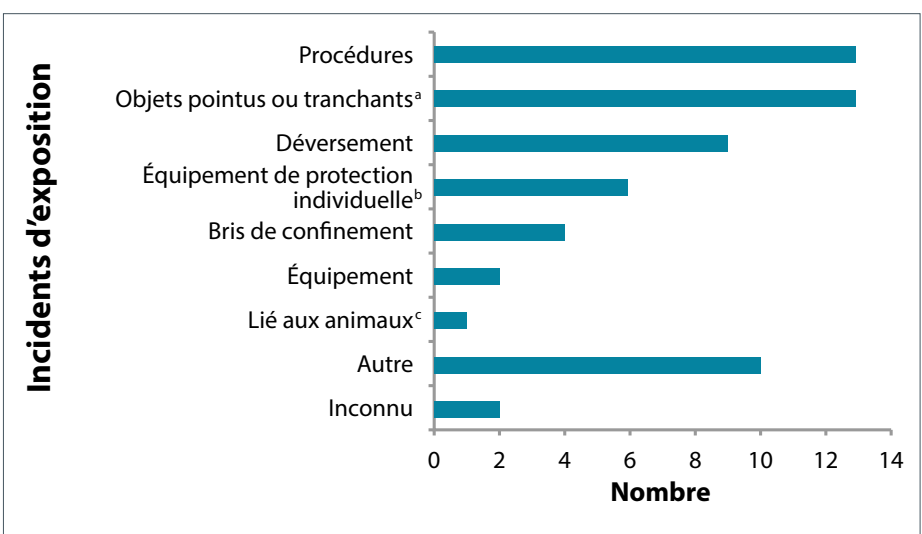

Abréviation : $\mathrm{N}$, nombre total

Note : Les données proviennent du système de surveillance de DILC (Canada, données extraites le 26 mars 2018)

a La catégorie " objets tranchants et piquants » comprend les piqûres d'aiguille et autres blessures par objets tranchants et piquants

b La catégorie " équipement de protection individuelle » comprend un équipement de protection individuelle inapproprié ou la défaillance d'un tel équipement

c La catégorie « animaux » comprend les morsures et les égratignures

\section{Voie d'exposition}

Parmi les 118 personnes touchées lors des 44 incidents, la majorité a pu être exposée à des matières infectieuses par inhalation ( $n=72 ; 61 \%$ ). La seconde voie d'exposition la plus courante était l'inoculation ou l'injection par piqûre d'aiguille ou par d'autres objets pointus ou tranchants ( $n=11 ; 9 \%)$. D'autres voies d'exposition comme l'absorption par contact avec la peau ou les muqueuses, l'inoculation par morsure ou par égratignure, ou l'ingestion ont aussi été rapportées dans un petit nombre de cas.

\section{Causes fondamentales}

En tout, 101 causes fondamentales ont été déterminées pour les 44 incidents déclarés en 2017, soit une moyenne de 2,3 causes fondamentales citées par incident. Le tableau 3 montre la distribution de chacune des causes fondamentales énumérées dans le rapport de suivi. Les PON ont été citées dans 37 rapports
Tableau 3 : Causes fondamentales des incidents d'exposition à un agent pathogène humain ou une toxine signalés au Canada en $2017(N=101)$

\begin{tabular}{|c|c|c|c|}
\hline \multirow{2}{*}{$\begin{array}{c}\text { Cause } \\
\text { fondamentale }\end{array}$} & \multirow{2}{*}{ Préoccupations } & \multicolumn{2}{|c|}{ Mentions } \\
\hline & & n & $\%$ \\
\hline \multirow{5}{*}{$\begin{array}{l}\text { Procédure } \\
\text { opérationnelle } \\
\text { normalisée (PON) }\end{array}$} & Documents connus, mais non suivis. & \multirow{5}{*}{37} & \multirow{5}{*}{84} \\
\hline & Documents non connus de l'utilisateur & & \\
\hline & Documents non suivis correctement & & \\
\hline & $\begin{array}{l}\text { Documents inappropriés pour la tâche ou } \\
\text { l'activité }\end{array}$ & & \\
\hline & $\begin{array}{l}\text { Documents non disponibles, mais auraient } \\
\text { dû l'être }\end{array}$ & & \\
\hline \multirow{3}{*}{ Interaction humaine } & $\begin{array}{l}\text { Amélioration nécessaire en ce qui } \\
\text { concerne l'étiquetage, l'installation, le } \\
\text { fonctionnement et la disposition des outils } \\
\text { ou de l'équipement }\end{array}$ & \multirow{3}{*}{14} & \multirow{3}{*}{32} \\
\hline & $\begin{array}{l}\text { Amélioration nécessaire en ce qui concerne } \\
\text { les facteurs environnementaux dans le lieu } \\
\text { de travail }\end{array}$ & & \\
\hline & $\begin{array}{l}\text { Amélioration nécessaire en ce qui concerne } \\
\text { les contraintes liées à la charge de travail, } \\
\text { au stress causé par la pression ou à d'autres } \\
\text { exigences }\end{array}$ & & \\
\hline \multirow{5}{*}{ Équipement } & $\begin{array}{l}\text { Amélioration nécessaire en ce qui concerne } \\
\text { la conception de l'équipement }\end{array}$ & \multirow{5}{*}{11} & \multirow{5}{*}{25} \\
\hline & Équipement mal entretenu & & \\
\hline & Défaillance de l'équipement & & \\
\hline & $\begin{array}{l}\text { Équipement non adapté à la tâche } \\
\text { effectuée }\end{array}$ & & \\
\hline & $\begin{array}{l}\text { Procédures de contrôle de la qualité non } \\
\text { respectées ou à améliorer }\end{array}$ & & \\
\hline \multirow{3}{*}{ Communication } & $\begin{array}{l}\text { Absence d'une méthode ou d'un système } \\
\text { de communication }\end{array}$ & \multirow{3}{*}{10} & \multirow{3}{*}{23} \\
\hline & Aucune information communiquée & & \\
\hline & $\begin{array}{l}\text { Information communiquée imprécise, } \\
\text { ambiguë ou incomprise }\end{array}$ & & \\
\hline \multirow{4}{*}{ Formation } & $\begin{array}{l}\text { Aucune formation élaborée ou mise en } \\
\text { œuvre }\end{array}$ & \multirow{4}{*}{8} & \multirow{4}{*}{18} \\
\hline & Formation inappropriée ou insuffisante & & \\
\hline & $\begin{array}{l}\text { Formation disponible, mais non suivie ou } \\
\text { inachevée }\end{array}$ & & \\
\hline & $\begin{array}{l}\text { Qualifications ou compétences insuffisantes } \\
\text { pour la tâche à exécuter }\end{array}$ & & \\
\hline \multirow{7}{*}{$\begin{array}{l}\text { Gestion et } \\
\text { supervision }\end{array}$} & $\begin{array}{l}\text { Amélioration nécessaire en ce qui concerne } \\
\text { la supervision }\end{array}$ & \multirow{7}{*}{7} & \multirow{7}{*}{16} \\
\hline & $\begin{array}{l}\text { Amélioration nécessaire en ce qui concerne } \\
\text { la vérification, I'évaluation et l'application } \\
\text { des procédures opérationnelles normalisées }\end{array}$ & & \\
\hline & $\begin{array}{l}\text { Amélioration nécessaire en ce qui concerne } \\
\text { la vérification, l'évaluation et la mise en } \\
\text { œuvre de la formation }\end{array}$ & & \\
\hline & $\begin{array}{l}\text { Amélioration nécessaire en ce qui concerne } \\
\text { la préparation }\end{array}$ & & \\
\hline & $\begin{array}{l}\text { Amélioration nécessaire en ce qui concerne } \\
\text { les facteurs humains }\end{array}$ & & \\
\hline & $\begin{array}{l}\text { Amélioration nécessaire en ce qui concerne } \\
\text { l'évaluation des risques }\end{array}$ & & \\
\hline & $\begin{array}{l}\text { Amélioration nécessaire en ce qui concerne } \\
\text { le recrutement du personnel }\end{array}$ & & \\
\hline \multicolumn{2}{|l|}{ Autre } & 14 & 32 \\
\hline
\end{tabular}

Abréviations : $N$, nombre total; $n$, nombre

Notes: Un incident peut être associé à plus d'une cause fondamentale; les pourcentages sont arrondis au chiffre entier le plus près

Les données proviennent du système de surveillance de DILC 
(84 \%), suivis de l'interaction humaine dans 14 rapports (32\%). L'équipement a aussi été mis en cause dans le quart ( $n=11$; $25 \%$ ) des incidents signalés.

\section{Discussion}

Globalement, la fréquence des expositions en laboratoire à des agents pathogènes et à des toxines au Canada demeure relativement faible en 2017, avec un total de 44 incidents à l'échelle du pays, ce qui représente un peu moins de $5 \%$ de l'ensemble des établissements titulaires de permis. La plupart des rapports de cas décrits avaient pour cause des procédures inadéquates ou des bris de procédure et des objets pointus ou tranchants. C'est la raison pour laquelle les procédures opérationnelles normalisées (PON) et les erreurs humaines ont été le plus souvent cités comme causes fondamentales des incidents.

Nous ne sommes pas en mesure de comparer ces résultats à ceux d'autres pays, puisqu'il n'existe aucun autre système de surveillance national comparable. Par exemple, aux États-Unis, la déclaration des incidents est limitée aux cas d'exposition à des agents pathogènes transmissibles par le sang.

La principale force de la présente étude réside dans le fait qu'elle repose sur la déclaration obligatoire et normalisée des incidents de laboratoire survenus au Canada, pour l'ensemble des toxines et des agents pathogènes réglementés. L'étude fournit donc un portrait global de la biosécurité dans tous les laboratoires titulaires de permis et permet d'évaluer la fréquence réelle des expositions et des ICL au Canada (10).

À l'inverse, la principale limite de cette étude découle de la possibilité que les données soient incomplètes. Dans certains cas, il arrive en effet que des incidents en laboratoire ne soient pas déclarés. Il est également possible que des incidents ne soient pas détectés ou ne soient tout simplement pas signalés en raison d'une méconnaissance ou d'une incompréhension des exigences en matière de déclaration, ou même d'une réticence à signaler ce genre d'événement, compte tenu de I'interprétation généralement négative du terme « incident » (9). Ce problème est en voie d'être réglé et, à mesure que les parties réglementées apprendront à mieux connaître et normaliseront les exigences en matière de déclaration, nous nous attendons à ce que la fréquence des déclarations augmente au cours des prochaines années.

L'information fournie dans les rapports de suivi peut aussi être biaisée en raison de la nature du processus d'enquête. Tenter de cerner les causes de l'incident en se fondant sur les symptômes ou les résultats généraux peut favoriser un biais de rappel dans les résultats de l'enquête. Pour réduire ces limites, le système de surveillance de DILC fait l'objet d'ajustements continuels dans le but d'améliorer la convivialité et la clarté de son interface et des formulaires. L'ASPC élabore actuellement des documents d'orientation pour appuyer les activités de déclaration des incidents et d'enquête des parties réglementées.

Des comparaisons préliminaires intéressantes peuvent être faites entre les données de 2016 et de 2017. Entre autres, même si le nombre d'incidents déclarés était censé augmenter au cours des premières années du système, il est passé de 46 en 2016 (11) à 44 en 2017. La fréquence des incidents d'exposition a diminué dans le secteur académique (de $35 \%$ en 2016 à $27 \%$ en 2017) et a augmenté dans le secteur hospitalier (de $26 \%$ en 2016 à $39 \%$ en 2017). Même si le nombre d'incidents d'exposition a diminué en 2017 , le nombre de personnes exposées a augmenté de $18 \%$, principalement en raison de l'incident ayant entraîné l'exposition de 29 personnes à Brucella suis. La proportion d'ABCSE a augmenté, passant de $24 \%$ en 2016 à $27 \%$ en 2017. En 2016 comme en 2017, les cas d'exposition liés à des procédures et à des objets pointus ou tranchants ont été les types d'incidents les plus cités; ces résultats concordent avec les résultats rapportés dans d'autres études $(9,12,13)$. Les déclarations faisant état de la possession accidentelle d'un agent pathogène humain du GR3 dans un laboratoire d'un niveau de confinement 2 ont été plus fréquentes en 2017 (32\%) qu'en 2016 (22\%). II convient toutefois de souligner qu'après seulement deux années de données complètes, il est trop tôt pour établir des données de référence fiables ou dégager des tendances.

L'information fournie dans ce rapport est importante pour plusieurs raisons. Elle donne une idée d'ensemble des pratiques actuelles en matière de biosécurité dans les laboratoires du Canada et des risques pour la biosécurité présents dans ces milieux, ce qui peut servir de base à l'analyse comparative d'autres programmes de déclaration, au Canada et ailleurs. L'ASPC a pu élaborer des activités visant à accroître la sensibilisation aux incidents qui surviennent couramment. Par exemple, elle a rédigé un bulletin d'information comprenant un avis traitant des blessures par objets pointus ou tranchants causées par des lames de scalpel jetables. Elle a aussi rédigé et publié dans une revue scientifique un article décrivant le risque d'erreurs d'identification des ABCSE du GR3 par spectrométrie de masse à désorption-ionisation laser assistée par matrice avec analyseur de temps de vol (SM-MALDI-TOF) (14).

\section{Conclusion}

La fréquence des incidents d'exposition survenus en laboratoire a été relativement faible en 2017. La voie d'exposition la plus courante était l'inhalation, tandis que les causes fondamentales les plus fréquentes étaient les problèmes relatifs aux $P O N$ et I'erreur humaine. Le système de surveillance de DILC continuera de déterminer les facteurs de risque et les défis récurrents liés à la biosécurité et à la biosûreté dans les laboratoires et de contribuer à l'excellence des enquêtes et des interventions associées aux incidents de laboratoire en partageant son expertise et les leçons apprises avec la communauté des laboratoires. 


\section{Déclaration des auteurs}

D. P. N. - Surveillance des incidents, analyse de données, rédaction - ébauche initiale, rédaction - révision et édition M. H. - Surveillance des incidents, rédaction - édition, supervision

F. T. - Surveillance des incidents, analyse de données, rédaction - édition, supervision

\section{Conflit d'intérêts}

Aucun.

\section{Remerciements}

Nous tenons à remercier Craig Brooks et Ken Turcotte pour leur expertise et leurs commentaires. Nous aimerions aussi remercier tous les titulaires de permis et les agents de la sécurité biologique pour la soumission de rapports d'une grande qualité.

\section{Financement}

Ce travail a été appuyé par l'Agence de la santé publique du Canada dans le cadre de son mandat de base.

\section{Références}

1. Reportable biosecurity incidents. Canberra: Department of Agriculture and Water Resources, Australian Government; 2017 [mise à jour le 17 août 2016]. http://www.agriculture. gov.au/biosecurity/legislation/reportable-incident

2. Health Protection Agency. Eye of the needle: United Kingdom Surveillance of Significant Occupational Exposures to Blood Borne Viruses in Healthcare Workers. London: Health Protection Agency; 2006. http://webarchive. nationalarchives.gov.uk/20140714113638/http://www.hpa. org.uk/webc/HPAwebFile/HPAweb_C/1205394781623

3. Electronic Code of Federal Regulations. n/a: Government Publishing Office; 2018 [mise à jour le 30 mai 2018]. Electronic Code of Federal Regulations. https://ecfr.io/

4. Gouvernement du Canada. Loi sur les agents pathogènes humains et les toxines (L.C. 2009, ch. 24), Obligation d'informer le Ministre. Ottawa (ON): Gouvernement du Canada; [mise à jour le 7 sept 2018] http://laws.justice.gc.ca/ fra/lois/H-5.67/page-2.html

5. Gouvernement du Canada. Site Web de la législation (Justice) : Règlement sur les agents pathogènes humains et les toxines (DORS/2015-44) : Loi sur les agents pathogènes humains et les toxines : Permis. Ottawa (ON): Gouvernement du Canada; [mise à jour le 7 sept]. http://laws.justice.gc.ca/ fra/reglements/DORS-2015-44/page-1.html
6. Agence de la santé publique du Canada. Guide canadien sur la biosécurité, Deuxième édition. Ottawa (ON): ASPC; 2016. https://www.canada.ca/fr/sante-publique/services/ normes-lignes-directrices-canadiennes-biosecurite/ guide-deuxieme-edition.html

7. List of Human Pathogens and Toxins for Export Control. Canberra (AU): Australia Group; 2017 [mise à jour en juill 2017]. http://www.australiagroup.net/en/human_animal_ pathogens.html

8. Ligne directrice canadienne sur la biosécurité. Notification et déclaration en vertu de la LAPHT et du RAPHT au moyen du module de déclaration du Portail de biosûreté. Ottawa (ON) : Agence de la santé publique du Canada; 2017 [mise à jour le 25 mai 2018]. https://www.canada.ca/fr/sante-publique/ services/normes-lignes-directrices-canadiennes-biosecurite/ directrices/notification-declaration-loi-reglement-agentspathogenes-humains-toxines.html

9. Bienek A, Heisz M, Su M. Surveillance des expositions en laboratoire aux agents pathogènes humains et aux toxines au Canada en 2016. Relevé des maladies transmissibles au Canada. 2017;43(11):259-68. DOI

10. Harding L, Byers K. Epidemiology of laboratory-associated infections. In: Flemming DO, Hunt DL, editors. Biological safety: principles and practices, $4^{\text {th }}$ edition. Washington (DC): ASM Press; 2006. p. 53-77.

11. Campbell MJ. Characterizing accidents, exposures, and laboratory-acquired infections reported to the National Institutes of Health's Office of Biotechnology Activities (NIH/OBA) Division under the NIH Guidelines for work with recombinant DNA materials from 1976-2010. Appl Biosaf 2015;20(1):12-26. DOI

12. De Carli G, Abiteboul D, Puro V. The importance of implementing safe sharps practices in the laboratory setting in Europe. Biochem Med (Zagreb) 2014 Feb;24(1):45-56. DOI PubMed

13. Sewell DL. Laboratory-associated infections and biosafety. Clin Microbiol Rev 1995 Jul;8(3):389-405. http://cmr.asm. org/content/8/3/389.short. PubMed

14. Pomerleau-Normandin D, Heisz M, Su M. Misidentification of risk group 3/Security Sensitive Biological agents by MALDI-TOF MS in Canada: November 2015-October 2017. Can Commun Dis Rep 2018;44(5):110-5. DOI

15. Agence de la santé publique du Canada. Norme canadienne sur la biosécurité. Deuxième édition. Ottawa (ON) : ASPC; 2015. https://www.canada.ca/fr/sante-publique/services/ normes-lignes-directrices-canadiennes-biosecurite/ deuxieme-edition.html 


\section{Annexe}

\section{Définitions liées à la Loi sur les agents pathogènes humains et les toxines}

\begin{tabular}{|c|c|}
\hline Terme & Définition \\
\hline Agent de la sécurité biologique (ASB) & $\begin{array}{l}\text { Personne désignée pour superviser les pratiques en matière de biosécurité et de biosûreté dans une } \\
\text { installation. }\end{array}$ \\
\hline Niveau de confinement (NC) & $\begin{array}{l}\text { Exigences minimales liées au confinement physique et aux pratiques opérationnelles visant la } \\
\text { manipulation sécuritaire d'agents pathogènes humains et de toxines dans les laboratoires. Il existe } \\
\text { quatre niveaux de confinement, qui vont du niveau de base au niveau le plus élevé (1 à } 4 \text { ). }\end{array}$ \\
\hline Zone de confinement & $\begin{array}{l}\text { Espace physique qui répond aux exigences liées à un niveau de confinement donné. Il peut s'agir } \\
\text { d'une salle unique, d'une série de salles situées dans un même endroit ou d'une série de salles } \\
\text { adjacentes. La zone de confinement peut comprendre des zones réservées au soutien, y compris } \\
\text { des antichambres, notamment des sas équipés de douches, de vestiaires " propres " et de vestiaires } \\
\text { "sales », le cas échéant. }\end{array}$ \\
\hline Exposition & $\begin{array}{l}\text { Contact ou proximité étroite avec des agents pathogènes humains ou des toxines pouvant } \\
\text { respectivement causer une infection ou une intoxication. Les voies d'exposition sont l'inhalation, } \\
\text { l'ingestion, l'inoculation et l'absorption. }\end{array}$ \\
\hline Formulaire de suivi de l'exposition & $\begin{array}{l}\text { Document utilisé pour rapporter et consigner des renseignements liés à une exposition accidentelle } \\
\text { préalablement déclarée à l'Agence de la santé publique du Canada, ainsi qu'à l'enquête qui y est } \\
\text { associée. }\end{array}$ \\
\hline Formulaire de notification de l'exposition & $\begin{array}{l}\text { Document utilisé pour déclarer une exposition accidentelle à l'Agence de la santé publique du Canada } \\
\text { et pour consigner les renseignements préliminaires associés à cette exposition. }\end{array}$ \\
\hline Incident & $\begin{array}{l}\text { Événement ou situation pouvant causer une blessure, un préjudice, une infection, une intoxication, } \\
\text { une maladie ou un dommage. Les incidents peuvent mettre en cause des matières infectieuses, des } \\
\text { animaux infectés ou des toxines. }\end{array}$ \\
\hline $\begin{array}{l}\text { Intoxication ou infection contractée en } \\
\text { laboratoire }\end{array}$ & $\begin{array}{l}\text { Intoxication ou infection résultant de l'exposition à des matières infectieuses, à des animaux infectés } \\
\text { ou à des toxines manipulés,ou entreposés dans la zone de confinement. }\end{array}$ \\
\hline Permis & $\begin{array}{l}\text { Autorisation délivrée par l'Agence de la santé publique du Canada en vertu de l'article } 18 \text { de la Loi } \\
\text { sur les agents pathogènes humains et les toxines, permettant de mener une ou plusieurs activités } \\
\text { réglementées comportant des agents pathogènes humains ou des toxines. Un permis peut s'appliquer } \\
\text { à plusieurs zones de confinement. }\end{array}$ \\
\hline Groupe de risque (GR) & $\begin{array}{l}\text { Groupe dans lequel les matières biologiques sont classées en fonction de leurs caractéristiques } \\
\text { inhérentes, comme la pathogénicité, la virulence, le risque de propagation et l'existence d'un } \\
\text { traitement prophylactique ou thérapeutique efficace. Le groupe de risque énonce le risque pour la } \\
\text { santé du personnel et du public ainsi que la santé des animaux et des populations animales. }\end{array}$ \\
\hline $\begin{array}{l}\text { Agents biologiques à cote de sécurité } \\
\text { élevée (ABCSE) }\end{array}$ & $\begin{array}{l}\text { Sous-ensemble d'agents pathogènes humains et de toxines qui présentent un risque accru en } \\
\text { matière de biosûreté, en raison de la possibilité qu'on les utilise comme arme biologique. Les agents } \\
\text { pathogènes humains et les toxines sont décrits comme étant " précisés par le règlement " à l'article } 10 \\
\text { du Règlement sur les agents pathogènes humains et les toxines. Les agents biologiques à cote de } \\
\text { sécurité élevée comprennent donc tous les agents pathogènes des groupes de risque } 3 \text { et } 4 \text { figurant } \\
\text { sur la Liste des agents pathogènes humains ou animaux et des toxines réglementés à l'exportation, } \\
\text { publiée par le Groupe d'Australie et sujette à modifications, à l'exception du virus Duvenhage, du virus } \\
\text { rabique et de toutes les autres espèces du genre Lyssavirus, du virus de la stomatite vésiculaire, ainsi } \\
\text { que du virus de la chorioméningite lymphocytaire. Les agents biologiques à cote de sécurité élevée } \\
\text { comprennent aussi toutes les toxines qui se trouvent à la fois à l'annexe } 1 \text { de la Loi sur les agents } \\
\text { pathogènes humains et les toxines et sur la Liste des agents pathogènes humains ou animaux et des } \\
\text { toxines réglementés à l'exportation et qui sont présentes en quantités supérieures aux quantités seuils } \\
\text { énoncées à l'article } 10 \text { (2) du Règlement sur les agents pathogènes humains et les toxines. }\end{array}$ \\
\hline
\end{tabular}

